\title{
COMPARATIVE EVALUATION OF EFFICACY OF GINGIVAL RETRACTION USING CHEMICAL AND MECHANICAL METHODS: AN IN VIVO STUDY
}

\section{PARAMPREET KAUR KOHLI, VEENA HEGDE*}

Department of Prosthodontics and Crown and Bridge, Manipal College of Dental Sciences, Manipal University, Manipal, Karnataka, India. Email: drveenahebbar@yahoo.com

Received: 19 September 2017, Revised and Accepted: 31 October 2017

\section{ABSTRACT}

Objective: The purpose of this in vivo study was to compare and evaluate the clinical efficacy of two gingival retraction systems; Ultrapak and Traxodent, on the basis of the amount of gingival retraction achieved in vertical and horizontal direction and their hemorrhage control.

Methods: A total of 60 subjects were selected requiring fixed prosthesis. The two gingival retraction systems were used on the prepared abutments randomly. The vertical gingival retraction was measured before and after retraction using flexible measuring strip with $0.5 \mathrm{~mm}$ grading. The horizontal retraction was measured on the casts poured in polysilicone impressions made before the retraction and after retraction.

Results: Statistically significant difference $(\mathrm{p}<0.05)$ was found between the amount of the retraction (vertical and horizontal) achieved by Ultrapak as compared to Traxodent. However, in achieving hemostasis Traxodent showed better efficiency than Ultrapak ( $<<0.05$ ).

Conclusion: The mean retraction width and depth achieved with retraction cord (Ultrapak) was significantly greater when compared with retraction paste. Although retraction paste (Traxodent) showed bleeding index significantly less when compared to that of retraction cord (Ultrapak).

Keywords: Gingival retraction, Ultrapak, Traxodent, Hemostatic control.

(C) 2018 The Authors. Published by Innovare Academic Sciences Pvt Ltd. This is an open access article under the CC BY license (http://creativecommons. org/licenses/by/4. 0/) DOI: http://dx.doi.org/10.22159/ajpcr.2018.v11i2.22674

\section{INTRODUCTION}

Marginal adaptation plays an important role in the long-term success of the restoration, and failure to achieve the same can result in ill-fitting crowns, hypersensitivity, marginal leakage, periodontal tissue inflammation, and increased risk of recurrent caries [1,2]. The process of gingival displacement allows the exposure of the gingival or subgingival finish line along with the adjacent unprepared part of the tooth [3].

At present, in the market, there are various methods of the gingival tissue management such as mechanical method (retraction cords), chemo-mechanical method (chemicals embedded in cords), and surgical method (lasers, electrosurgery, and rotary curettage), of which gingival retraction cords are most commonly used $[4,5]$.

The use of retraction cords can generate decent retraction, but clinicians usually report with the problem of gingival trauma and the time taken in the placement of the cord. Furthermore, there have been various investigations into the tendency of displacement cords to encourage bleeding and cause acute injury, which usually takes more than 1 week to heal. Gingival manipulation may result in significant bleeding in those patients taking antiplatelet medications [6-8] and those with preexisting periodontal diseases [9]. Hence, the retraction material should not only displace the gingival tissue laterally and vertically but also control the bleeding [1,10-12].

Recently, cordless systems have been developed to save time and enhance patient compliance. The material offered is usually paste or foam that is injected into the crevicular sulcus $[1,13,14]$. This removes the need for the clinician to physically compress the material into the sulcus, where it may generate high pressure and cause injury.

The newly introduced Traxodent ${ }^{\circledR}$ hemodent paste retraction material (Premier Dental Products Co.) comprises 15\% aluminum chloride topical paste and cotton caps, have been designed to improve the gingival displacement and assist in hemostasis.

Thus, the aim of the following study was to compare the efficiency of the gingival cord (mechanical) and Traxodent (chemical) in achieving the horizontal and vertical displacement of the gingival tissue along with its hemostatic potential.

\section{METHODS}

A total of 60 patients were selected for this study. Before the study, the protocol was explained to the participants, and informed consent was obtained. The study protocol was approved by the Institutional Ethical Committee. The sample size was further divided into two equal groups of 30 each, Group A and Group B.

1. Group A (30 patients): Gingival retraction cord Ultrapak 000 was used the mechanical method.

2. Group B (30 patients): Traxodent ${ }^{\circledR}$ retraction material was used the chemical method.

The inclusion criteria for the study included:

1. Any posterior (premolars and molars) edentulous area requiring a fixed partial denture.

2. Age $>18$ years.

3. Clinically and radiographically healthy abutment tooth.

4. Abutment teeth of normal size and contour (no development anomaly or regressive changes).

5. Probing depth $<3 \mathrm{~mm}$.

While the exclusion criteria were:

1. Age $<18$ years.

2. Signs of attachment loss and clinical mobility.

3. Presence of exudates.

4. Uncontrolled diabetes, hypertension, hyperthyroidism, and other cardiovascular disorders. 
Before starting the crown preparation, alginate impression was made for the selected arch to fabricate the customized sectional tray for making an elastomeric impression. This was followed by a veneer crown preparation where the finish line was placed equigingivally. After this, an elastomeric impression was taken with the help of addition polysilicon using the customized sectional tray using double mix single impression technique. The impression was checked for any voids, following which it was boxed and then poured in diestone. The cast retrieved was used to check for the horizontal retraction.

Then a smooth, flexible measuring strip with $0.5 \mathrm{~mm}$ grading was used to measure the sulcular depth in the vertical direction at mesiobuccal, midbuccal, and distobuccal region before the retraction was performed (Fig. 1 and 2). Next, the gingiva around the abutment tooth was retracted either with the help of Ultrapak (Group A) or Traxodent (Group B).

\section{Retraction with the retraction cord (Ultrapack 000)}

After measuring the sulcular depth, sufficient amount of retraction cord was cut and looped around the preparation. With the help of the cord packer, it was gently tucked down beneath the finish line of the preparation (Fig. 3). It was left in the place for $5 \mathrm{~min}$.

Then, the cord was removed, and the preparation was air-dried. The hemostatic potential was checked, and accordingly, scores 0,1 , and 2 were given in Table 1.

\section{Retraction with traxodent}

The prepared tooth was air-dried, and it was made sure that the margins were kept dry. The paste was dispensed with the help of a syringe into the gingival sulcus. The paste was slowly applied into the sulcus all around the teeth (Fig. 4). The whitening of the gingiva showed that the paste was well applied. The paste was left there for 2 min (according to manufacturer's guidelines) and then rinsed off with water. Next, the tooth surface was air-dried, and hemostatic potential was checked, and accordingly, scores 0,1 , and 2 were given in Table 1 .

After the retraction procedure (both in Group A and Group B) sulcular depth in the vertical direction using flexible strip ( $0.5 \mathrm{~mm}$ gradient) was measured in mesiobuccal, midbuccal, and distobuccal region, and post-retraction elastomeric impression was made, and the cast was retrieved.

The cast obtained (both pre- and post-retraction) was checked under the stereomicroscope (Fig. 5), and horizontal retraction was checked in mesiobuccal, midbuccal, and the distobuccal region (Fig. 6).

Table 1: Hemorrhage scores

\begin{tabular}{ll}
\hline Score 0 & No bleeding \\
Score 1 & Bleeding controlled within $1 \mathrm{~min}$ \\
Score 2 & Bleeding not controlled within $1 \mathrm{~min}$ \\
\hline
\end{tabular}

Table 2: Mean values and standard deviations of horizontal and vertical retraction achieved in Group A and Group B $(n=30)$

\begin{tabular}{lllll}
\hline Direction & Site & Mean \pm SD & \multirow{2}{*}{$\mathbf{p}$} \\
\cline { 3 - 4 } & & Group A & Group B & \\
\hline Horizontal & Mesiobuccal & $0.248 \pm 0.038$ & $0.230 \pm 0.031$ & 0.055 ; NS \\
& Midbuccal & $0.264 \pm 0.035$ & $0.233 \pm 0.025$ & $<0.001$; Sig \\
& Distobuccal & $0.271 \pm 0.043$ & $0.236 \pm 0.023$ & $<0.001$; Sig \\
\multirow{5}{*}{ Vertical } & Average & $0.261 \pm 0.033$ & $0.233 \pm 0.019$ & $<0.001$; Sig \\
& Mesiobuccal & $1.017 \pm 0.334$ & $0.600 \pm 0.193$ & $<0.001$; Sig \\
& Midbuccal & $1.008 \pm 0.331$ & $0.617 \pm 0.205$ & $<0.001$; Sig \\
& Distobuccal & $0.975 \pm 0.343$ & $0.558 \pm 0.157$ & $<0.001$; Sig \\
& Average & $1.000 \pm 0.295$ & $0.592 \pm 0.157$ & $<0.001$; Sig \\
\hline
\end{tabular}

Sig: Significant, SD: Standard deviation, NS: Non significant

\section{Data analysis}

Statistical analysis was performed using SPSS version 18. $p<0.05$ was considered statistically significant. Comparison of mean scores for horizontal and vertical retraction was done using independent sample t-test, and Mann-Whitney U test was used to compare the hemostatic score.

\section{RESULTS}



Fig. 1: Flexible measuring strip



Fig. 2: Measurement of the sulcular depth

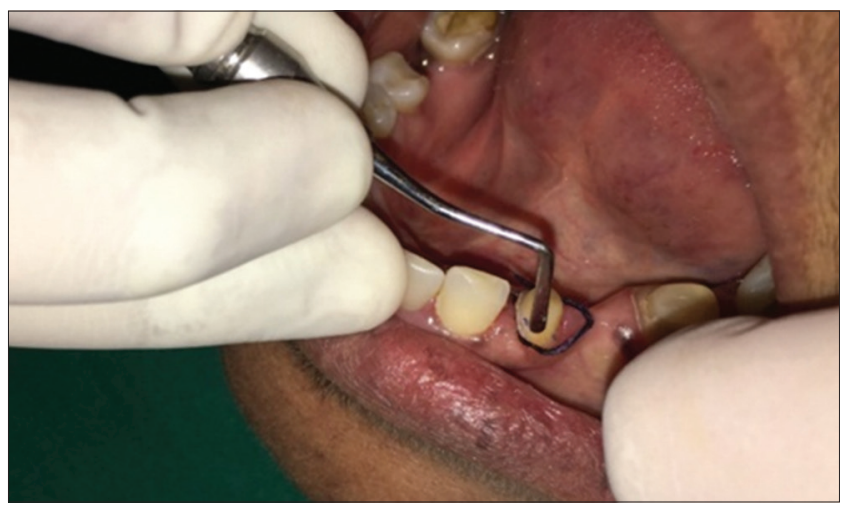

Fig. 3: Placement of the cord



Fig. 4: Application of the Traxodent 
The mean horizontal and vertical retraction achieved by different retraction systems are listed in Table 2 . The comparison of mean scores for horizontal and vertical retraction was done using independent sample t-test, and it showed a significant difference $(\mathrm{p}<0.05)$ in the amount of retraction achieved.

The hemorrhage scores on removal of each retraction system were compared using Mann-Whitney U test in Table 3 and the results showed a significant difference $(\mathrm{p}<0.05)$.

The Ultrapak retraction cord induced maximal bleeding on removal, while Traxodent induced minimal bleeding on removal.

\section{DISCUSSION}

Clinical parameters such as the location of the finish line, periodontal health of the patient along with sulcular bleeding during the process of impression directly influence the quality of the impression making in fixed partial prosthodontics. To obtain accurate marginal fit of the prosthesis a precise transfer from the patient to the definitive cast is essential. Hence, gingival displacement is necessary not only to record the gingival finish line but also the adjacent tooth structure [15]

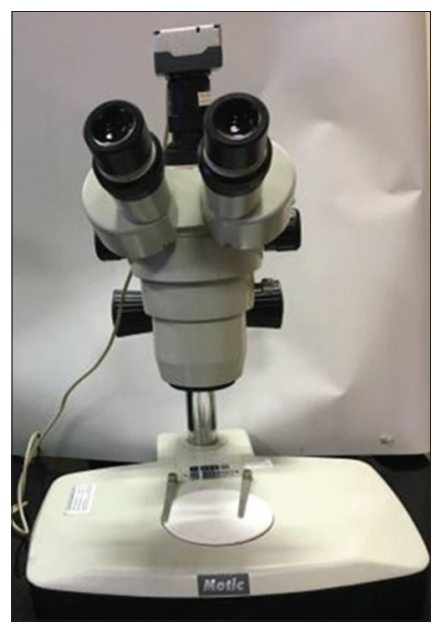

Fig. 5: Stereomicroscope

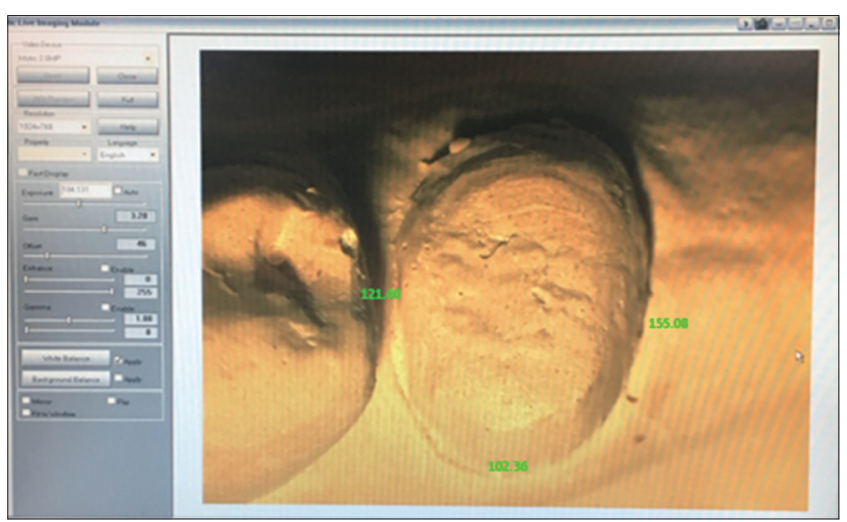

Fig. 6: Cast examined under the stereomicroscope

Table 3: Mean values and standard deviations of the hemostatic score of Group A and Group B

\begin{tabular}{lcc}
\hline \multicolumn{2}{c}{ Mean \pm SD } & p \\
\cline { 1 - 2 } Group A & Group B & \\
\hline $1.73 \pm 0.45$ & $0.17 \pm 0.38$ & $<0.001 ;$ Sig \\
\hline Sig: Significant, SD: Standard deviation
\end{tabular}

All the measurements made in the study were performed by single operator to avoid the inter-operator variability. The results obtained from this study can be attributed to the fact that Ultrapak cord is a mean of a mechanical method of gingival retraction and clinicians have to physically compress the cord into the sulcus to obtain the maximum gingival retraction [16]. On the other hand, Traxodent is a chemical method where no physical pressure was applied while placing it into the gingival sulcus. Hence, the amount of retraction achieved was less for Traxodent as compared to the Ultrapak. This result is similar to that in the study by Gupta et al. [16] in the year 2013. In this study, statistically significant differences were found between the horizontal and vertical retraction achieved by Ultrapak as compared to Traxodent. However, this finding is contrary to the results reported by et al.[17] who found that gingival displacement paste exhibited enhanced response in attaining horizontal displacement of the gingival sulcus as compared to the gingival retraction cord.

Based on the results attained, Ultrapak showed maximum bleeding on removal, while Traxodent encouraged no bleeding on removal. A study conducted by Acar et al. in the year 2014 [1] evaluated the clinical performance and the impression quality between the cordless, and conventional retraction system showed that the displacement paste and cap showed better results in terms of ease of application, time spent and bleeding. Furthermore, the pressure generated by the cordless system was studied by Bennani et al. [4] in the year 2014 confirmed atraumatic pressure, with lower levels of post-treatment inflammatory cytokines as compared to the cord system.

Although utmost care was taken to avoid any source of human error in the present study, some errors are inevitable that may have occurred during the retraction of gingival tissues, in the process of impression making and while pouring the experimental casts.

In this study, only one variety of mechanical and chemical retraction agents were chosen. Further studies should be conducted to test different variety of retraction systems available commercially and manufactured specifically for fixed partial impressions. Other retraction techniques such as double cord technique were not considered only single retraction cord technique was followed while using Ultrapak in all the cases. To standardize the variables and to minimize the errors, the net amount of vertical and horizontal retraction was considered. In addition, some variation may have been caused by the flexible measuring strips due to the difference in the compressibility of the gingival tissues. In future studies can be conducted using digital technique instead of measuring it manually. The major drawback of the study being that the influence of distensibility of gingiva, the gingival thickness, varied sulcus depth, location of the abutment teeth (posterior maxillary or mandibular), and the visibility and accessibility on the gingival retraction were not taken into the consideration.

\section{CONCLUSION}

This clinical study investigated the retraction efficiency and hemostatic potential of the cord and cordless system of gingival retraction. The mean retraction width and depth achieved with retraction cord (Ultrapak) was significantly greater when compared with retraction paste (Traxodent), while retraction paste showed bleeding index significantly less when compared to the retraction cord.

\section{AUTHORS CONTRIBUTION}

Parampreet Kohli-Compiled and analysed the data. Veena HegdeAnlyzed the data and prepared the manuscript.

\section{CONFLICTS OF INTERESTS}

None.

\section{REFERENCES}

1. Acar O, Erkut S, Ozcelik TB, Ozdemir E, Akcil M. Comparison of 
cordless and conventional displacement system regarding clinical performance and impression quality. J Prosthet Dent 2014;111:388-94.

2. Felton DA, Kanoy BE, Bayne SC, Wirthman GP. Effect of in vivo crown margin discrepancies on periodontal health. J Prosthet Dent 1991;65:357-64

3. Chandra S, Singh A, Gupta KK, Chandra C, Arora V. Effect of gingival displacement cord and cordless systems on the closure, displacement, and inflammation of the gingival crevice. J Prosthet Dent 2016;115:177-82.

4. Bennani V, Inger M, John M. Arts comparison of pressure generated by cordless gingival displacement materials. J Prosthet Dent 2014;112:163-7.

5. Al-Ani A, Bennani V, Chandler NP, Lyons KM, Thomson WM. New Zealand dentists' use of gingival retraction techniques for fixed prosthodontics and implants. N Z Dent J 2010;106:92-6.

6. Madhulaxmi M, Wahab PU. Can aspirin be continued during dental extraction? Int J Pharm Pharm Sci 2014;6:20-3.

7. Kumar S. Local hemostatic agents in the management of bleeding in oral surgery. Asian J Pharm Clin Res 2016;9:35-41.

8. Kumar S. Dental management of patients on antiplatelet therapy: Literature update. Asian J Pharm Clin Res 2016;9:26-31.

9. Neha MM, Kishore NG. Orodental delivery systems: An overview. Int J Pharm Pharm Sci 2013;5:74-83.

10. Donovan TE, Gandara BK, Nemetz H. Review and survey of medicaments used with gingival retraction cords. J Prosthet Dent 1985;53:525-31

11. Baharav H, Kupershmidt I, Laufer BZ, Cardash HS. The effect of sulcular width on the linear accuracy of impression materials in the presence of an undercut. Int J Prosthodont 2004;17:585-9.

12. Johnson GH, Mancl LA, Schwedhelm ER, Verhoef DR, Lepe X. Clinical trial investigating success rates for polyether and vinyl polysiloxane impressions made with full-arch and dual-arch plastic trays. J Prosthet Dent 2010;103:13-22.

13. Al Hamad KQ, Azar WZ, Alwaeli HA, Said KN. A clinical study on the effects of cordless and conventional retraction techniques on the gingival and periodontal health. J Clin Periodontol 2008;35:1053-8.

14. Yang JC, Tsai CM, Chen MS, Wei JY, Lee SY, Lin TL. Clinical study of a newly developed injection-type gingival retraction material. Chin Dent J 2005;24:147-51.

15. Cooper K, Bennani V, Tawse-Smith A, Reid M, Dias GS. Effect of a cordless retraction paste on titanium surface: A topographic, chemical and biocompatibility evaluation. Braz Oral Res 2013;27:211-7.

16. Gupta A, Prithviraj DR, Gupta D, Shruti DP. Clinical evaluation of three new gingival retraction systems: A research report. J Indian Prosthodont Soc 2013;13:36-42.

17. Prasanna GS, Reddy K, Kumar RK, Shivaprakash S. Evaluation of efficacy of different gingival displacement materials on gingival sulcus width. J Contemp Dent Pract 2013;14:217-21 